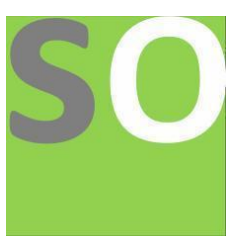

Article title: An improved bound on the sum of prime numbers

Authors: Monica Feliksiak [1]

Affiliations: N.A.[1]

Orcid ids: 0000-0002-5786-4227[1]

Contact e-mail: monicafeliksiak@yahoo.com

License information: This work has been published open access under Creative Commons Attribution License http://creativecommons.org/licenses/by/4.0/, which permits unrestricted use, distribution, and reproduction in any medium, provided the original work is properly cited. Conditions, terms of use and publishing policy can be found at https://www.scienceopen.com/.

Preprint statement: This article is a preprint and has not been peer-reviewed, under consideration and submitted to ScienceOpen Preprints for open peer review.

DOI: 10.14293/S2199-1006.1.SOR-.PPD7MQS.v1

Preprint first posted online: 09 July 2021

Keywords: R. Mandl inequality; sum of primes Supremum; sum of primes Estimate. 


\title{
AN IMPROVED BOUND ON THE SUM OF PRIME NUMBERS.
}

\author{
MONICA U. FELIKSIAK
}

\begin{abstract}
.
We derive two asymptotic formulae for the upper bound on the sum of the first $\mathrm{n}$ primes. Both the Supremum and the Estimate of the sum are superior to known bounds. The Estimate bound had been derived to promote efficiency of estimation of the sum.
\end{abstract}

2010 Mathematics Subject Classification. 1102, 1103 11A41, 11K65, 11L20, 11N05, 11N37.

Key words and phrases. R. Mandl's inequality, sum of primes Supremum, sum of primes estimate. 


\section{ACKNOWLEDGEMENT}

I am very grateful to my father J. Feliksiak, who extended his help by providing many hints for me and assisting with revision. I thank him for his generosity and time spent.

M. U. Feliksiak 
1. The Sum of Prime Numbers: The R. Mandl's inequality.
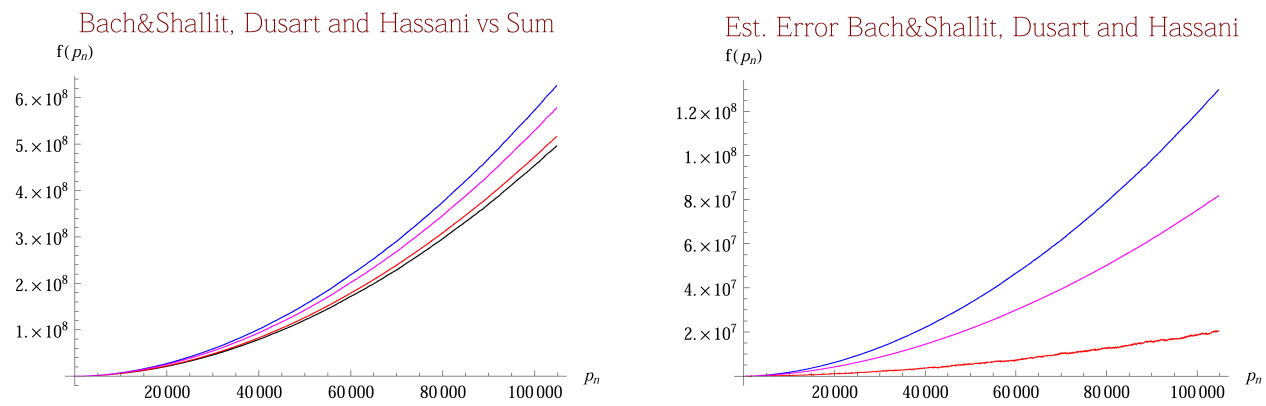

Figure 1. The drawing on the left compares the graphs od Dussart's (in blue), Bach \& Shallit (in purple) and Hassani's (in red) estimates as compared to the true sum of primes (in black). The drawing on the right compares the estimation error made by the respective estimates.

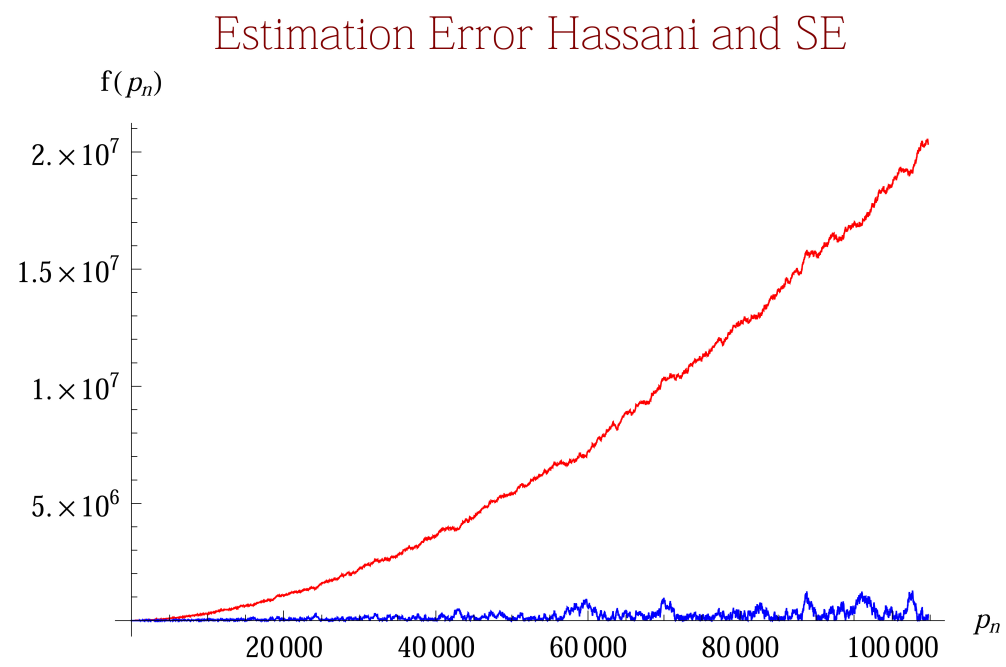

Figure 2. The drawing compares the estimation error made by the Hassani's estimate (in red) and the absolute error made by the Sharp Estimate S.E. (in blue)

The conjectured inequality by R. Mandl, has been proven by J.B. Rosser and L. Schoenfeld in their 1975 paper [8]. The Mandl's inequality asserts that:

$$
\sum_{i=1}^{n} p_{i}<\frac{n \times p_{n}}{2}
$$


The efforts directed to refine and sharpen the Mandl's estimate include for example Dusart [2] who proved that:

$$
\sum_{i=1}^{n} p_{i} \leq \frac{n^{2}}{2}\left(\log n+\log \log n-\frac{3}{2}+\frac{\log \log n-2.29}{\log n}\right) \quad \forall n \geq 10134
$$

M. Hassani [7] in 2006 presented a considerably improved refinement:

$$
\sum_{i=1}^{n} p_{i}<\frac{n \times p_{n}}{2}-\frac{n^{2}}{14} \quad \forall n \geq 10
$$

These estimates however, diverge relatively quickly from the actual sum of the prime numbers. In contrast to that, theory based on the Gauss' offset integral provides for a significantly improved bounds. Let's therefore define:

Definition 1.1 (True sum of prime numbers).

$\mathcal{T} . \mathcal{S} .=\sum_{i=1}^{n} p_{i}$

Definition 1.2 (Supremum Upper Integration Limit).

$$
\theta_{(s)}=2 \sum_{i=1}^{n}\left(p_{i} \log p_{i}\right)
$$

Definition 1.3 (Sharp Estimate $\mathcal{S} \mathcal{E}_{{ }_{\left(p_{n}\right)}}$ Upper Integration Limit).

$$
\theta_{(e)}=\left(p_{n}-(\sqrt{5}-1)\left(4 \gamma^{2}-2 \gamma\right)\left(\log p_{(n)}\right) \sqrt[3]{p_{(n)}}\right)^{2}
$$

and $\gamma \approx 0.57721566490153286$ is the Euler-Mascheroni constant.

Definition 1.4 (Absolute Estimation Error of the $\mathcal{S} \cdot \mathcal{E} \cdot\left(p_{n}\right)$ ).

$$
\mathcal{E} \cdot \mathcal{E} \cdot{ }_{\left(p_{n}\right)}=\left|\left(\mathcal{S} \cdot \mathcal{E} \cdot{ }_{\left(p_{n}\right)}=\int_{2}^{\theta_{(e)}} \frac{d t}{\log t}\right)-\sum_{i=1}^{n} p_{i}=\mathcal{T} \cdot \mathcal{S} .\right| \quad \forall p_{n} \in \mathbb{N} \mid p_{(n)} \geq 2
$$

In this section we present the Supremum $\mathcal{S U P}_{\left(p_{n}\right)}$ upper bound, as well as the Sharp Estimate $\mathcal{S} \cdot \mathcal{E} \cdot\left(p_{n}\right)$. Both these functions depend on the theory of the primorial function and its bounds, presented here:

Lemma 1.5 (Upper Bound on the logarithm of the primorial).

The natural logarithm of the primorial function is strictly less than the respective prime number $p \in \mathbb{N}$ :

(1.4) $\log p_{(n)} \sharp<p_{(n)} \leq n \quad \forall n \in \mathbb{N} \mid n \geq 2$, where $p_{n}$ is the largest prime $p \leq n$ In particular the natural logarithm of the primorial function is asymptotic (from below) to the respective prime number:

$$
\log p_{(n)} \sharp \sim p_{(n)}
$$

Lemma 1.6 (Lower Estimation Error Bound On The Difference $\left.p_{n}-\log p_{n} \sharp\right)$.

The error of estimation of the primorial function by the use of the value of $p_{(n)}$ imposes the following lower bound:

$$
\begin{gathered}
\mathcal{L B}_{p_{(n)}}=(\sqrt{5}-1)\left(4 \gamma^{2}-2 \gamma\right)\left(\log p_{(n)}\right) \sqrt[3]{p_{(n)}}<\left(p_{(n)}-\log p_{(n)} \sharp\right) \\
\forall p_{(n)} \in \mathbb{N} \mid p_{(n)} \geq 2
\end{gathered}
$$

where $\gamma \approx 0.57721566490153286060651209$ is the Euler-Mascheroni constant. 
Lemma 1.7 (Upper Estimation Error Bound On The Difference $\left.p_{n}-\log p_{n} \sharp\right)$.

The error of estimation of the primorial function by the use of the value of $p_{(n)}$ imposes the following upper bound:

$$
\left(p_{(n)}-\log p_{(n)} \sharp\right)<2 \sqrt{p_{(n)}}=\mathcal{U} \mathcal{B}_{p_{(n)}} \quad \forall p_{(n)} \in \mathbb{N} \mid p_{(n)} \geq 2
$$

For the proofs of Lemmas 1.5, 1.6 and 1.7, please consult Feliksiak [5].

\subsection{Supremum Bound on the Sum of Prime Numbers.}

The Supremum bound and the Sharp Estimate, both implement a variant of the Gauss' offset logarithmic integral. The Supremum is a very accurate bound, its computation however will become challenging very quickly. The degree of computational difficulty of the Supremum is about the same as computing the sum itself. Consequently, the Sharp Estimate $\mathcal{S} \mathcal{E}_{\cdot\left(p_{n}\right)}$ is so to say, the "golden mean" to achieve the goal.

The Sharp Estimate $\mathcal{S} \mathcal{E}_{\cdot\left(p_{n}\right)}$ is quick and straightforward to compute. Thus, it attenuates the computational difficulty, while at the same time it is sufficiently accurate to rely on. The $\mathcal{S} \cdot \mathcal{E}_{\cdot\left(p_{n}\right)}$ cuts through the meanders of the True Sum of the prime numbers, producing alternatively positive/negative estimation error values. By taking the Absolute value of the Estimation Error, we can demonstrate that the estimation error, at the prime $p_{n}=19373$ already falls below 1 percent of the value that the True Sum attains. The estimation error improves as $p_{n} \rightarrow \infty$. The Figures 3 and 4 serve as a visual aid and are not a part of the proofs.
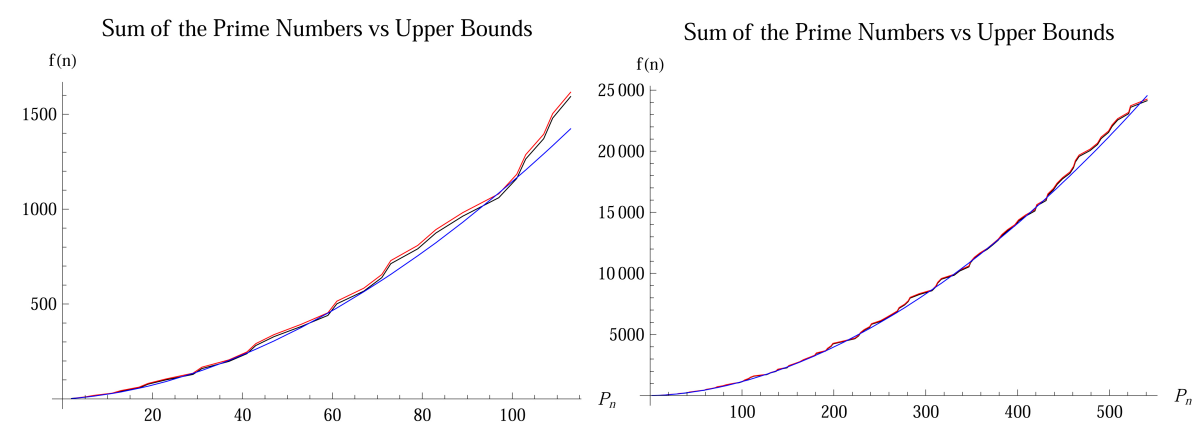

Figure 3. The drawings show the graphs of the True Sum of primes (in black), the Supremum bound (in red) and the Sharp Estimate S.E. (in blue). Figures are drawn w.r.t. $p_{n}$. 

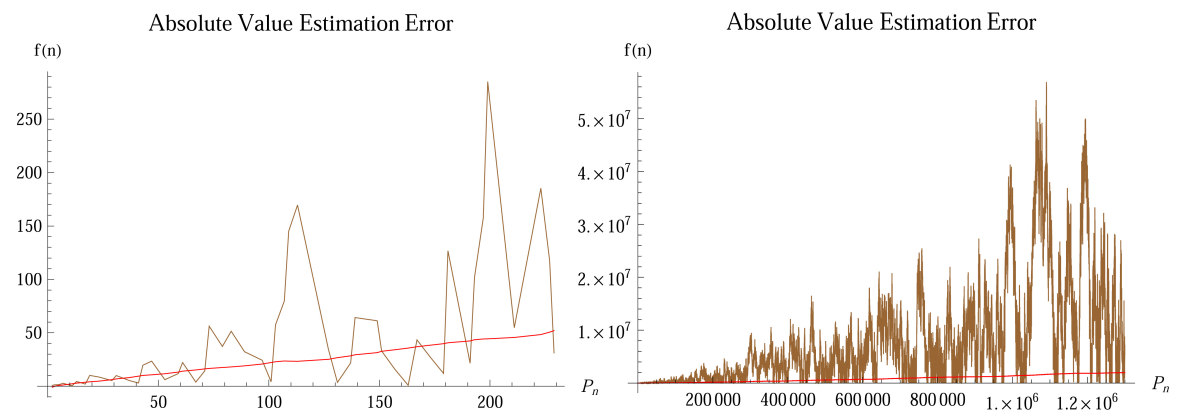

Figure 4. The drawings contrast the graphs of the estimation error made by the Supremum bound (in red) and the absolute estimation error made by the Sharp Estimate S.E. (in brown). Figures are drawn w.r.t. $p_{n}$.

Lemma 1.8 (The Supremum Bound on the Sum of Prime Numbers).

The Supremum bound on the sum of primes up to $p_{n}$, for all $p_{n} \in \mathbb{N} \mid p_{n} \geq 5$ is given by:

$$
\mathcal{S U P}_{p_{n}}=\int_{2}^{\theta_{s}} \frac{d t}{\log t}>\sum_{i=1}^{n} p_{(i)}=\mathcal{T} \mathcal{S} \text {. }
$$

Where $\mathcal{T} . \mathcal{S}$. and $\theta_{s}$ are given by the Definitions: 1.1 and 1.2 respectively.

Proof.

Suppose that for $p_{n} \in \mathbb{N} \mid p_{n} \geq 17$ the inequality is false:

$$
\left[\left(\left(\int_{2}^{\theta_{s}} \frac{d t}{\log t}\right) /\left(\sum_{i=1}^{n} p_{(i)}\right)\right)-1\right]>\frac{1}{p_{n}}
$$

This implies that:

$$
\left[\left(\left(\int_{2}^{\theta_{s}} \frac{d t}{\log t}\right) /\left(\sum_{i=1}^{n} p_{(i)}\right)\right)-1\right]^{(-1)}>p_{n}
$$

However, at $p_{n}=17$ the difference of terms of the inequality 1.10 attains approximately -1.3563587 and diverges at every step $n$, at the rate $\propto k p_{n}$ with $k \rightarrow 1$ as $p_{n} \rightarrow \infty$. Consequently, we have a contradiction. Necessarily therefore,

$$
\left[\left(\left(\int_{2}^{\theta_{s}} \frac{d t}{\log t}\right) /\left(\sum_{i=1}^{n} p_{(i)}\right)\right)-1\right]^{(-1)}<p_{n}
$$

for all $p_{n} \in \mathbb{N} \mid p_{n} \geq 17$ the inequality 1.11 holds. This in turn implies that for all $p_{n} \in \mathbb{N} \mid p_{n} \geq 17$

$$
\left(\int_{2}^{\theta_{s}} \frac{d t}{\log t}\right)>\sum_{i=1}^{n} p_{(i)}\left(1+\frac{1}{p_{n}}\right)=\left(\sum_{i=1}^{n} p_{(i)}+\frac{\sum_{i=1}^{n} p_{(i)}}{p_{n}}\right)
$$

Consequently, Lemma 1.8 holds for all $p_{n} \in \mathbb{N} \mid p_{n} \geq 17$. For all $5 \leq p_{n} \leq 17$ Table 1 lists all values of $p_{n}$ within the range, demonstrating that Lemma 1.8 holds in this 
range as well. Thus, necessarily Lemma 1.8 holds as stated for all $p_{n} \in \mathbb{N} \mid p_{n} \geq 5$, concluding the proof.

TABle 1. Low Range Difference of $\mathcal{S U P}_{p_{n}}$ and $\mathcal{T} . \mathcal{S}$.

\begin{tabular}{|c|c|c|c|}
\hline$p_{n}$ & $\mathcal{S U} \mathcal{P}_{p_{n}}-\mathcal{T} . \mathcal{S}$. & $p_{n}$ & $\mathcal{S U} \mathcal{P}_{p_{n}}-\mathcal{T} . \mathcal{S}$. \\
\hline 3 & -0.159632 & 11 & 2.25869 \\
\hline 5 & 0.609876 & 13 & 2.81551 \\
\hline 7 & 1.10942 & 17 & 3.70758 \\
\hline
\end{tabular}

\subsection{Sharp Estimate of the Sum of Primes.}

Definition 1.9 (Sharp Estimate $\mathcal{S} \cdot \mathcal{E} \cdot{ }_{\left(p_{n}\right)}$ of the Sum of Prime Numbers).

$$
\mathcal{S} \cdot \mathcal{E} \cdot{ }_{\left(p_{n}\right)}=\int_{2}^{\theta_{(e)}} \frac{d t}{\log t} \quad \forall p_{n} \in \mathbb{N} \mid p_{(n)} \geq 2
$$

Lemma 1.10 (Bounds on Relative Error of the Sharp Estimate $\mathcal{S} \cdot \mathcal{E} \cdot\left(p_{n}\right)$ ).

The absolute relative error, of the Sharp Estimate of the sum of prime numbers up to $p_{n}$, for all $p_{n} \in \mathbb{N} \mid p_{n} \geq 2$ has the following bounds:

$$
\mathcal{L} \cdot \mathcal{B} \cdot_{\left(p_{n}\right)}=\frac{1}{\left(p_{n}\right)^{2}}<\mathcal{R} . \mathcal{E} \cdot{ }_{\left(p_{n}\right)}=\frac{|\mathcal{E} \cdot \mathcal{E} \cdot|}{\mathcal{T} \cdot \mathcal{S} .}<\frac{1}{\sqrt[3]{p_{n}}}=\mathcal{U} \cdot \mathcal{B} \cdot_{\left(p_{n}\right)}
$$

Where $\mathcal{S} \cdot \mathcal{E} \cdot\left(p_{n}\right), \theta_{e}$ and $\mathcal{E} . \mathcal{E}$. are given by the Definitions $1.9,1.3$ and 1.4 respectively While $\gamma \approx 0.57721566490153286061$ is the Euler-Mascheroni constant.

Proof.

Suppose that for $p_{n} \in \mathbb{N} \mid p_{n} \geq 2$, the following inequality is false:

$$
\frac{\left|\left[\left(\int_{2}^{\theta_{e}} \frac{d t}{\log t}\right)-\mathcal{T} . \mathcal{S} .\right]\right|}{\mathcal{T} . \mathcal{S} .}-\frac{1}{\left(p_{n}\right)^{2}}>0
$$

This implies that it must be true:

$$
\left(\frac{\left|\left[\left(\int_{2}^{\theta_{e}} \frac{d t}{\log t}\right)-\mathcal{T} . \mathcal{S} .\right]\right|}{\mathcal{T} \mathcal{S} .}\right)^{(-1)}-\left(p_{n}\right)^{2}>0
$$

However, at $p_{n}=2$ the inequality 1.16 attains $\sim-0.910865$ and diverges rapidly at every step, at a rate $\propto p_{n}^{2}$. Consequently we have a contradiction to the initial hypothesis. Necessarily therefore, for all $p_{n} \in \mathbb{N} \mid p_{n} \geq 2$ :

$$
\frac{\left|\left[\left(\int_{2}^{\theta_{e}} \frac{d t}{\log t}\right)-\mathcal{T} . \mathcal{S} .\right]\right|}{\mathcal{T} . \mathcal{S} .}>\frac{1}{\left(p_{n}\right)^{2}}
$$

the inequality 1.18 must be true. Now suppose, that for $p_{n} \in \mathbb{N} \mid p_{n} \geq 127$ the following inequality is false:

$$
\frac{\left|\left[\left(\int_{2}^{\theta_{e}} \frac{d t}{\log t}\right)-\mathcal{T} . \mathcal{S} .\right]\right|}{\mathcal{T} . \mathcal{S} .}-\frac{1}{\sqrt[3]{p_{n}}}<0
$$


This implies that:

$$
\left(\frac{\left|\left[\left(\int_{2}^{\theta_{e}} \frac{d t}{\log t}\right)-\mathcal{T} . \mathcal{S} .\right]\right|}{\mathcal{T} . \mathcal{S} .}\right)^{(-1)}-\left(\sqrt[3]{p_{n}}\right)<0
$$

However, at $p_{n}=127$ inequality 1.19 attains $\sim 45.218$, and diverges at every step, at a rate exceeding $\sqrt[3]{p_{n}}$. The divergence rate further increases as $p_{n}$ increases unboundedly. Consequently we have a contradiction to the initial hypothesis. Thus necessarily, for all $p_{n} \in \mathbb{N} \mid p_{n} \geq 127$ :

$$
\frac{\left|\left[\left(\int_{2}^{\theta_{e}} \frac{d t}{\log t}\right)-\mathcal{T} \cdot \mathcal{S} .\right]\right|}{\mathcal{T} . \mathcal{S} .}<\frac{1}{\sqrt[3]{p_{n}}}
$$

Direct computer evaluation demonstrates that for $p_{n}$ in the range $2 \leq p_{n} \leq 127$, inequality 1.20 holds. The output in graphical form is given in Figure 5. Thus Lemma 1.10 holds as stated. Concluding the proof.

Corollary 1.11 (The Relative Error $\mathcal{R}$.E. $_{\left(p_{n}\right)}$ of $\mathcal{S}$.E $_{\cdot_{\left(p_{n}\right)}}$ Limit).

Clearly, both the lower bound $\mathcal{L} \cdot \mathcal{B} \cdot{ }_{\left(p_{n}\right)}$ as well as the upper bound $\mathcal{U} \cdot \mathcal{B} \cdot\left(p_{n}\right)$ converge to zero as $p_{n}$ increases unboundedly. Consequently this implies, that the relative error $\mathcal{R} \mathcal{E}_{\cdot\left(p_{n}\right)}$ of $\mathcal{S} \mathcal{E}_{\cdot\left(p_{n}\right)}$ given by Lemma 1.10 , which is squeezed between those bounds, must converge to zero as well:

$$
\lim _{p_{n} \rightarrow \infty}(\mathcal{L B})=\lim _{p_{n} \rightarrow \infty}\left(\left|\mathcal{R} \cdot \mathcal{E} \cdot{ }_{\left(p_{n}\right)}\right|\right)=\lim _{p_{n} \rightarrow \infty}(\mathcal{U B}) \rightarrow 0
$$
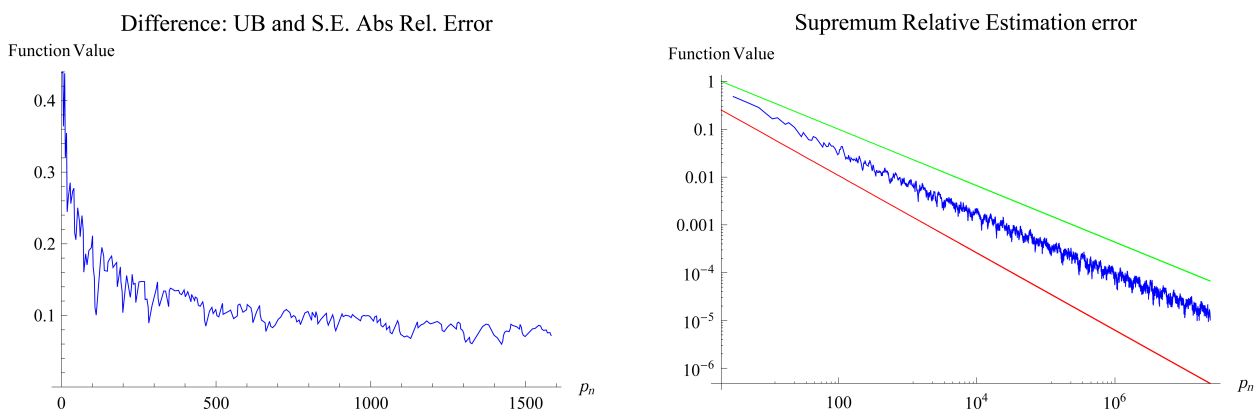

Figure 5. The linear drawing on the left shows the difference of terms of the $\mathcal{U} \cdot \mathcal{B} \cdot{ }_{\left(p_{n}\right)}$ and $\mathcal{R} . \mathcal{E} \cdot{ }_{\left(p_{n}\right)}$, the figure is drawn w.r.t. $p_{n} \in \mathbb{N}$. The log-log drawing on the right shows the Relative Estimation Error $\mathcal{R} . \mathcal{E} \cdot{ }_{\left(p_{n}\right)}$ (in blue), the lower bound $\mathcal{L} \cdot \mathcal{B} \cdot{ }_{\left(p_{n}\right)}$ (in green) and the upper bound $\mathcal{U} \cdot \mathcal{B} \cdot{ }_{\left(p_{n}\right)}$ (in red) over the range $p_{n} \in$ $\mathbb{N} \mid 2 \leq p_{n} \leq 23879519$. 


\section{REFERENCES}

[1] R. Crandall and C. Pomerance, Prime numbers, a computational perspective, Springer Verlag, New York, 2005.

[2] P. Dusart, Autour de la fonction qui compte le nombre de nombres premiers, Limoges, 1998.

[3] Paul Erdös, On the difference of consecutive primes, Quarterly Journal Of Mathematics (1935).

[4] Paul Erdös and E.G. Strauss, Remarks on the differences between consecutive primes, Elem. Math. 35 (1980), 115-118.

[5] Jan Feliksiak, The elementary proof of the Riemann's Hypothesis, MDPI, AG, https://doi.org/10.20944/preprints202006.0365.v1 (2020).

[6] G.H. Hardy and E.M. Wright, An introduction to the theory of numbers, Oxford University Press, London, 1968.

[7] M. Hassani, A refinement of Mandl's inequality and approximation of the product $p_{1} p_{2} \cdots p_{n}$, arXiv:math/0606765v1[math.NT] (2006).

[8] J.B. Rosser and L. Schoenfeld, Sharper bounds for the Chebyshev functions $\theta_{x}$ and $\psi_{x}$, Mathematics of Computation 29 (1975), no. 129, 243-269.

[9] Sebastian M. Ruiz, A result on prime numbers, Mathematical Gazette 81 (1997), no. 269, 269.

[10] Daniel Shanks, On maximal gaps between successive primes, Math. Comp. 18 (1964), 646651. 


\section{Appendix}

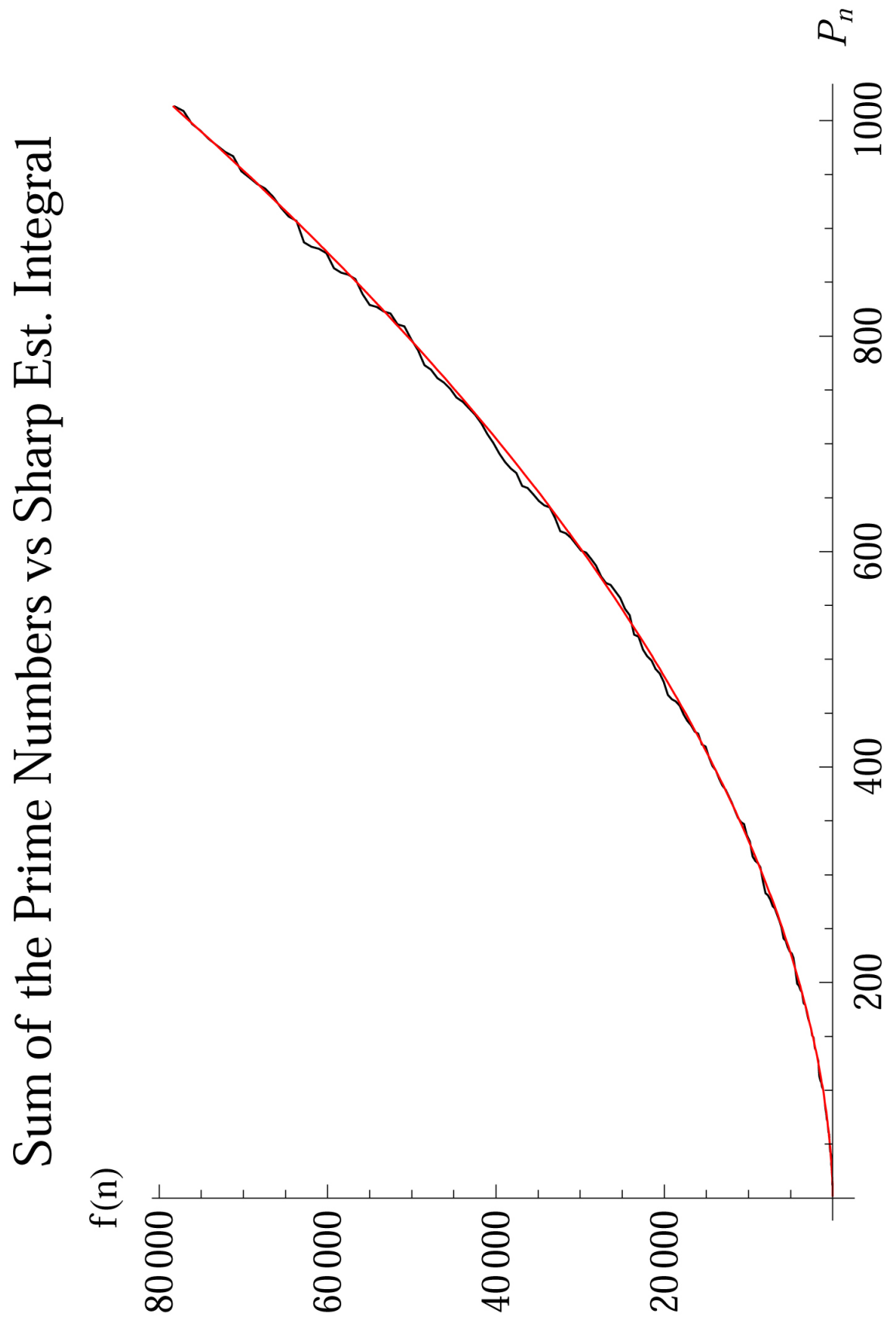

Figure 6. The drawing shows the graphs of the True Sum of primes (in black) and the Sharp Estimate S.E. (in red) w.r.t. $p_{n}$. 